\title{
190 SPORTS PSYCHOLOGY IN INDIA
}

Chaitanya Sridhar Jain University, Bangalore, Karnataka, India

10.1136/bjsm.2010.078725.190

Consider the following scenario - a highly talented player on whom rests a million hopes is well prepared physically and dreaming of gold. But then just at the start of the match, he realises he hasn't learnt to deal with his nerves (and thoughts), which are essential to focus and perform. Now is it solely the players fault that he didn't know how to manage the psychological aspects of the game? It is a well established fact that mental skills training is an integral component to achieve peak performance. Why then is sports psychology still in its infancy in India? Countries like Australia and USA have a sport psychologist attached to every team. Why it is still not accepted in our country? One of the main reasons could be the negative connotation associated with psychology. Till date many 
a players and coaches think going to a psychologist means there is a 'problem'. Some of the other reasons are the lack of resource personnel, lack of awareness, following traditional methods of coaching with little emphasis on sport psychology and sports science. Also, 'image management' is a key issue in India, especially in sports. Players want to maintain 'I'm cool and have no problem attitude'. Mental skills training ought to be treated as a component of peak performance akin to physical training. Only, then can we think of achievement. What then is the solution? An integrative approach involving coaching with psychology and sports science is the "need of the hour'. 